Rojas-Luna, E. \& Santa-Cruz-Espinoza, H. (2021). El rol del docente ante situaciones de agresividad en el aula. Revista Electrónica Interuniversitaria de Formación del Profesorado, 24(3), 73-86.

DOI: https://doi.org/10.6018/reifop.466151

\title{
El rol del docente ante situaciones de agresividad en el aula
}

Edwin Rojas Luna, Henry Santa-Cruz-Espinoza.

Universidad César Vallejo, Trujillo, Perú.

\section{Resumen}

La conducta agresiva es una de las problemáticas de mayor incidencia entre los escolares, y debido a sus consecuencias sobre el clima escolar ha causado alarma en los docentes, quienes tienen como tarea realizar acciones con el fin de prevenirla o disminuirla, para ello es necesario dotarlos de herramientas que apoyen su identificación. Con este fin, el estudio tuvo como objetivos determinar las evidencias de validez del Cuestionario de Agresividad Premeditada e Impulsiva (CAPI-A), así como describir ambos tipos de agresividad en una muestra de 528 escolares peruanos entre 12 a 18 años. El análisis del instrumento evidencia validez de contenido ( $v o>, 70$ ), de estructura interna ( $R S M E A=, 064, C F I=, 96, T L I=, 95)$ y convergente $(r>, 30)$; así como adecuada confiabilidad y estabilidad temporal $(\omega>, 70$; testrestest $>, 70)$. En el estudio se encuentran también diferencias significativas $(p<, 001)$ en la agresividad premeditada e impulsiva en función del género, obteniéndose puntuaciones mayores en los varones. Se concluye que el CAPI-A es un instrumento que muestra evidencias de validez y confiabilidad, y se recomienda como instrumento en el proceso de identificación y toma de decisiones en torno a la conducta agresiva.

\section{Palabras clave}

Agresión; validez; confiabilidad; escolares; género. 


\title{
The role of the teacher in situations of aggressiveness in the classroom
}

\begin{abstract}
Aggressive behavior is one of the problems with the highest incidence among schoolchildren, and due to its consequences on the school climate it has caused alarm in teachers, who have the task of taking actions in order to prevent or reduce it, for this it is necessary to equip them of tools that support their identification. To this end, the study aimed to determine the evidence of validity of the Premeditated and Impulsive Aggression Questionnaire (CAPI-A), as well as to describe both types of aggressiveness in a sample of 528 Peruvian schoolchildren between 12 and 18 years old. The analysis of the instrument shows validity of content (vo>, 70), of internal structure (RSMEA =.064, $\mathrm{CFI}=, 96, \mathrm{TLI}=, 95)$ and convergent ( $r>$, 30); as well as adequate reliability and temporal stability ( $\omega>, 70$; test-restest>, 70). The study also found significant differences $(p<.001)$ in premeditated and impulsive aggressiveness as a function of gender, obtaining higher scores in males. It is concluded that the CAPI-A is an instrument that shows evidence of validity and reliability, and it is recommended as an instrument in the process of identification and decision-making regarding aggressive behavior.
\end{abstract}

\section{Key words}

Aggression; validity; reliability; schoolchildren; gender.

\section{Introducción}

Los centros educativos persiguen el propósito de posibilitar el proceso de enseñanzaaprendizaje, promoviendo el desarrollo integral del grupo de alumnos; empero, en ciertas circunstancias este proceso puede modificarse por la manifestación de conductas violentas (Álvarez-García et al., 2010). Hoy, la violencia escolar se ha conformado como un problema de salud relevante que sucede en diversas modalidades (McMahon et al., 2019). Desde sus primeros estudios, las cifras de violencia escolar incrementaron a lo largo del mundo (Martínez Martínez et al., 2017). La agresividad en las aulas aparece en etapas escolares cada vez más precoces, con mayor frecuencia en la educación secundaria (Pinazo et al., 2020).

Estadísticas mundiales indican que la mitad de jóvenes sufren de violencia entre compañeros de escuela, y uno de cada tres estudiantes, entre 13 y 15 años, se involucró en peleas físicas en su colegio (Fondo de las Naciones Unidas para la Infancia [Unicef], 2018). Asimismo, la Organización de las Naciones Unidas para la Educación, la Ciencia y la Cultura (Unesco, 2018) notifica que uno de cada tres educandos fueron víctimas de Bullying, y tres de cada 10, aceptan que acosaron a sus compañeros en algún momento.

En el aula de clases, la conducta agresiva sucede de diferentes formas, desde situaciones en apariencia menores como esconder cosas de los compañeros, poner apodos, molestar; a acciones críticas como robos, destrozos, amenazas con armas, agresiones físicas, entre otros; hecho que implican consecuencias negativas: por ejemplo, un clima escolar alterado (Álvarez-García et al., 2010). Igualmente, un estudio sugiere que el comportamiento agresivo de los compañeros puede escalar la agresión en estudiantes que no presentaban comportamientos de esa naturaleza (Thomas et al., 2011). En dicho sentido, son los maestros los agentes capacitados para conseguir un cambio, descendiendo comportamientos agresivos en el ambiente familiar y escolar (Cuenca Sánchez y Mendoza González, 2017). 
Un aspecto relevante para el estudio de estas conductas, parte de su conceptualización; empero, hay dificultad cuando se procura definir este constructo ya que engloba una amplia gama de conductas que dificultan concentrarlo en un solo concepto (Andreu Rodríguez, Ramírez et al., 2006). Por un lado, se definió a la agresividad como una acción destructiva y dañina encaminada a generar daño a otro (Andreu, Peña y Ramírez, 2009). También puede ser comprendida como una interacción entre el agresor y la víctima, donde el agresor posee el impulso de generar daño al agredido revelando emociones como ira o frustración; en ciertos casos, puede estar dirigida al ejercicio de defender una posición, rango social o fuente de poder (Andreu, 2010).

La conducta agresiva es percibida como un fenómeno social y un problema psicológico (Farnicka y Grzegorzewska, 2015). La aparición de conductas agresivas en el contexto escolar, no puede ser atribuido a una solo causa, sino más bien a un conjunto de causas que interactúan entre sí. Se halló que los problemas de externalización en adolescentes se vinculan a estilos parentales inconsistentes (Jiménez-Barbero et al., 2016), como el estilo parental autoritario (Ruíz-Hernández et al., 2019). Asimismo, poca afectividad o sobreprotección parental (Boboc y Rosoiu, 2019) podrían repercutir en esta problemática. Igualmente, está relacionado al uso de redes sociales (Martín Montilla et al., 2016), a un nivel más bajo de habilidades sociales de empatía y autocontrol (Caballero et al., 2018) y a conductas delictivas (Zuiev, 2016).

Como se observa, la agresión es un comportamiento social complejo con diversas causas y manifestaciones. Sucede de forma física mediante golpes; o verbal, por intermedio de gritos o insultos; asimismo, su naturaleza puede ser directa o indirecta (Denson, O'Dean, Blake y Beames, 2018). En vista de ello, la investigación sugiere diferencias de género en las formas de comportamiento agresivo, pero no existe consenso al respecto. En algunos casos, se cree que, en mayor medida, son los hombres quienes presentan mayores niveles de agresividad (Muñoz-Reyes et al., 2016; Perez Bernardes de Moraes, 2013). Empero, al contrastar las formas de agresividad, se identifica que tanto mujeres y hombres son verbalmente igual de agresivos; en tanto que los varones evidencian más agresividad física que las mujeres (Björkqvist, 2018).

Por otro lado, ciertos autores informan dos tipos de conducta agresiva: premeditada (CAP) e impulsiva (CAl), basada en el criterio de funcionalidad motivacional, equiparada a otra dicotomía denominada reactiva y proactiva. El análisis de la agresividad impulsiva condujo a distinguir ciertos aspectos peculiares como su respuesta no planificada, derivada de la ira cuya motivación es dañar a la víctima como resultado de la provocación percibida. Por su parte, la agresión premeditada está dirigida a la obtención de un objetivo diferente al de dañar a la víctima, asimismo emplea estrategias agresivas para resolver conflictos (Garaigordobil et al., 2017).

Por todo lo mencionado, es necesario que el docente cuente con la posibilidad de identificar conductas agresivas en el aula de clases, cuente con instrumentos válidos y fiables que le ayuden en esa labor. En ese sentido, y luego de una exhaustiva revisión bibliográfica, este estudio consideró el Cuestionario de Agresividad Premeditada e Impulsiva en Adolescentes (CAPI-A) como un instrumento adecuado para ser usado en el contexto escolar, puesto que estudios antecesores respaldan sus propiedades psicométricas. Remontándonos a su historia, en el 2006, Raine et al. construyeron el Reactive-Proactive Aggression Questionnaire (RPQ) de 23 ítems, que posteriormente fue adaptada por Andreu et al. (2009) en una muestra de 732 adolescentes de la comunidad de Madrid. Se identificó validez de estructura interna con cargas factoriales aceptables y consistencia interna de ,91 de la escala total, ,84 de agresividad reactiva y ,87 de agresividad proactiva, enfocándose en dos manifestaciones de la agresión, aportando a la comprensión de la conducta agresiva en ámbitos clínicos y no 
clínicos, sirviendo para el diagnóstico de algunos problemas de comportamiento, y establecer parámetros e indicadores en la producción de estrategias de intervención.

Posteriormente, en función del modelo de los dos factores sobre CAP y CAI, Andreu (2010) creó el Cuestionario de Agresividad Premeditada e Impulsiva en Adolescentes (CAPI-A) validado en adolescentes madrileños $(n=825)$, consiguiendo evidencias aceptables en la revisión de la estructura ( $A F C$ ), hallándose una confiabilidad de, 83 en la dimensión de CAP, y ,82 en la dimensión de CAl; además se evaluó la validez convergente, determinando correlaciones significativas con el RPQ y la Escala de Impulsividad de Barrat.

En el contexto peruano, no se realizaron estudios importantes que evalúen las propiedades psicométricas de un instrumento que posibilite la identificación de la conducta agresiva en escolares, incluso cuando la comprensión de estas conductas puede servir de inicio para el planteamiento de intervenciones que ayuden a mejorar la vida de los adolescentes y de la sociedad (Socastro-Gómez y Jiménez, 2019), lo que no ha permitido tampoco tener una descripción objetiva de la agresión en los adolescentes. En tal sentido, se establecieron como objetivos determinar la evidencia de validez basada en el contenido, de la estructura interna, validez convergente y confiabilidad del CAPI-A en adolescentes escolarizados; asimismo, comparar ambos tipos de agresividad en función del género. Todo ello con el propósito de contribuir a la identificación y posterior intervención de la conducta agresiva en la esfera educativa.

\section{Metodología}

\section{Diseño y participantes}

Este estudio se clasifica como trasversal e instrumental, porque se realiza en un solo momento temporal determinado y analiza las propiedades psicométricas de los instrumentos (Ato et al., 2013)

Se utilizó un muestreo no probabilístico por conveniencia, con una muestra conformada por 528 adolescentes, 280 varones y 248 mujeres, del nivel secundario de un distrito peruano, con edades comprendidas entre los 12 a 18 años $(M=15,45$ y $D S=1,83)$.

\section{Instrumentos}

Cuestionario de Agresividad Premeditada e Impulsiva

EI CAPI-A, bajo un modelo bidimensional-dicotómico con las dimensiones: CAP, medida por los ítems 2, 4, 6, 8, 10,12, 14, 16, 18, 20, 22, 23, 24, orientada a evaluar la reacción proactiva, las creencias de eficacia positiva, el actuar de forma instrumental y la agresión direccionando con un objetivo; y b) CAl, medida por los ítems 1, 3, 5, 7, 9, 11, 13, 15, 17, 19, 21, orientada a evaluar la afectación emocional negativa, tendencia hostil, respuesta ante una provocación y reacción reactiva. Con un total de 24 ítems, se le atribuyó una escala tipo Likert de 5 puntos, donde 1 significa estar muy desacuerdo y 5 significa estar muy de acuerdo. Además, sobre las propiedades psicométricas, de acuerdo al análisis de su estructura a través del método de General Least Squares se obtuvo: GFI = ,87, AGFI = ,85, señalando que el modelo empírico ajusta al modelo teórico, además se encontró cargas factoriales desde ,30, que advierten una explicación de más del 10\% de la variabilidad de los ítems. Respecto a la confiabilidad, CAI reportó un valor de $\alpha=, 82$ y CAP alcanzó el valor de $a=, 83$ (Andreu, 2010).

Reactive/Proactive Questionnaire (RPQ) 
El cuestionario desarrollado en el 2006 por Raine et al., posee un modelo bifactorial conformado por los factores: agresión reactiva y agresión proactiva. Consta de 23 ítems con tres opciones de respuesta en una escala de Likert. Sus propiedades psicométricas fueron estudiadas en una muestra de estudiantes peruanos. El análisis de su estructura interna mediante el método de estimación de mínimos cuadrados diagonalmente ponderados robustos (DWLS) mostró índices adecuados de ajuste para el modelo de dos factores (CFI= ,982; TLI= ,980; RMSEA = 053[,051; ,056]; SRMR= ,058). La estimación de la fiabilidad se dio mediante los coeficientes de $a$ de Cronbach y $\omega$ de McDonald, obteniendo en la dimensión agresión reactiva $(\alpha=, 826 ; \omega=, 828)$ y agresión proactiva $(\alpha=, 852 ; \omega=, 863)$ adecuados indicadores de confiabilidad (Rojas Zegarra et al., 2020).

\section{Procedimientos}

Inicialmente el instrumento fue revisado por 12 profesionales con un formato de evaluación elaborado por los autores. Posteriormente, se solicitó la autorización para administrar el CAPI-A. Obtenido el permiso, se administró la evaluación en grupos de 25 estudiantes en sus aulas de clase, con un tiempo de 30 minutos aproximadamente para cada uno de ellos. En conformidad con los procedimientos éticos, se solicitó el asentimiento y la carta de testigo informado. Recolectada la información usando la técnica de la encuesta se revisaron las pruebas y codificaron con números correlativos, haciendo uso de una base de datos en el software Excel 2016 y procediendo al análisis estadístico.

\section{Análisis de datos}

Utilizando el método de juicio de expertos con la información obtenida de 15 profesionales se analizó la validez de contenido (Sireci y Faulkner-Bond, 2014) centrando el análisis en la claridad, coherencia y relevancia de los ítems en función al modelo teórico se obtuvieron las medidas V de Aiken en el software Excel 2016 e intervalos de confianza de cada ítem. Luego, se estudió la validez de estructura vaciando los datos a un libro de Excel (2016) y procesando los datos en el software estadístico R 3.1.6. de Lavaan. Se obtuvo las medidas de resumen media, desviación estándar, asimetría y curtosis para analizar la normalidad de los datos. Enseguida, se procedió a la revisión de la estructura usando el método de mínimos cuadrados ponderados robustos [DWLS], a razón del nivel de medición de las variables que corresponde a variables ordinales (Brown, 2006). Se estimaron los índices de ajuste: $\mathrm{x}^{2} / \mathrm{gl}$ (valores entre 2 y 5) (Escobedo Portillo et al., 2016), RSMEA $(<0,08)$, S-RMR $(\leq, 06), \operatorname{CFI}(\geq 0,95)$ y TLI $(\geq 0,95)$ (Ruíz et al., 2010); a fin de corroborar la idoneidad de la estructura, en complemento a la medición de las cargas factoriales. La validez convergente fue estudiada con el RPQ, utilizando el coeficiente de correlación de Pearson, tomando en cuenta las medidas de tamaño de efecto. Luego, se calculó la confiabilidad a partir del coeficiente de omega y el estadístico $r$ de Pearson (Ventura-León, 2018), pretendiendo determinar cuán precisa es la medida del CAPI-A. Finalmente, para realizar la comparación de la agresividad, según al género de los participantes, se utilizó el estadístico $U$ de Mann Whitney, debido a la distribución asimétrica de los datos.

\section{Resultados}

\section{Validez de contenido}

En la tabla 1, se presenta la revisión de expertos $\left(n_{\text {jueces }}=12\right)$, quienes valoraron la relevancia, coherencia y claridad de los ítems utilizando el coeficiente $V$ de Aiken. Reportaron índices desde .94 a 1 en relevancia, .92 a 1 en coherencia y en claridad; y el límite inferior del intervalo de confianza sugirió que el criterio era aceptable (vo > ,70). Los 24 ítems demostraron ser enunciados representativos para la medición de la agresividad premeditada e impulsiva. 
Tabla 1.

Índices $\mathrm{V}$ de Aiken de los ítems del cuestionario

Nota: V: Coeficiente V de Aiken; IC: Intervalos de confianza

\begin{tabular}{|c|c|c|c|c|c|c|c|c|c|}
\hline \multirow{3}{*}{$\begin{array}{c}\text { Ítems } \\
1\end{array}$} & \multicolumn{3}{|c|}{ Relevancia } & \multicolumn{3}{|c|}{ Coherencia } & \multicolumn{3}{|c|}{ Claridad } \\
\hline & \multirow{2}{*}{$\frac{V}{0,97}$} & \multicolumn{2}{|c|}{ IC 95\% } & \multirow{2}{*}{$\frac{V}{0,97}$} & \multicolumn{2}{|c|}{ IC $95 \%$} & \multirow{2}{*}{$\frac{V}{1,00}$} & \multicolumn{2}{|c|}{ IC 95\% } \\
\hline & & 0,86 & 1,00 & & 0,86 & 1,00 & & 0,90 & 1,00 \\
\hline 2 & 1,00 & 0,90 & 1,00 & 1,00 & 0,90 & 1,00 & 1,00 & 0,90 & 1,00 \\
\hline 3 & 0,94 & 0,90 & 1,00 & 1,00 & 0,90 & 1,00 & 0,97 & 0,86 & 1,00 \\
\hline 4 & 0,97 & 0,86 & 1,00 & 0,97 & 0,86 & 1,00 & 0,97 & 0,86 & 1,00 \\
\hline 5 & 0,94 & 0,82 & 0,98 & 0,94 & 0,82 & 0,98 & 0,97 & 0,86 & 1,00 \\
\hline 6 & 0,94 & 0,82 & 0,98 & 1,00 & 0,90 & 1,00 & 0,92 & 0,78 & 0,97 \\
\hline 7 & 0,97 & 0,86 & 1,00 & 0,97 & 0,86 & 1,00 & 0,94 & 0,82 & 0,98 \\
\hline 8 & 1,00 & 0,90 & 1,00 & 1,00 & 0,90 & 1,00 & 1,00 & 0,90 & 1,00 \\
\hline 9 & 0,97 & 0,86 & 1,00 & 0,97 & 0,86 & 1,00 & 1,00 & 0,90 & 1,00 \\
\hline 10 & 1,00 & 0,90 & 1,00 & 1,00 & 0,90 & 1,00 & 0,92 & 0,78 & 0,97 \\
\hline 11 & 0,97 & 0,86 & 1,00 & 0,97 & 0,86 & 1,00 & 0,94 & 0,82 & 0,98 \\
\hline 12 & 0,97 & 0,86 & 1,00 & 0,97 & 0,86 & 1,00 & 0,92 & 0,78 & 0,97 \\
\hline 13 & 0,97 & 0,86 & 1,00 & 0,97 & 0,86 & 1,00 & 0,94 & 0,82 & 0,98 \\
\hline 14 & 0,92 & 0,78 & 0,97 & 0,92 & 0,78 & 0,97 & 0,92 & 0,78 & 0,97 \\
\hline 15 & 1,00 & 0,90 & 1,00 & 1,00 & 0,90 & 1,00 & 1,00 & 0,90 & 1,00 \\
\hline 16 & 0,94 & 0,82 & 0,98 & 0,97 & 0,86 & 1,00 & 1,00 & 0,90 & 1,00 \\
\hline 17 & 0,97 & 0,86 & 1,00 & 0,97 & 0,86 & 1,00 & 1,00 & 0,90 & 1,00 \\
\hline 18 & 0,97 & 0,86 & 1,00 & 0,97 & 0,86 & 1,00 & 0,97 & 0,86 & 1,00 \\
\hline 19 & 0,92 & 0,78 & 0,97 & 0,97 & 0,86 & 1,00 & 1,00 & 0,90 & 1,00 \\
\hline 20 & 1,00 & 0,90 & 1,00 & 1,00 & 0,90 & 1,00 & 1,00 & 0,90 & 1,00 \\
\hline 21 & 0,94 & 0,82 & 0,98 & 0,97 & 0,86 & 1,00 & 0,97 & 0,86 & 1,00 \\
\hline 22 & 0,97 & 0,86 & 1,00 & 0,97 & 0,86 & 1,00 & 0,94 & 0,82 & 0,98 \\
\hline 23 & 0,97 & 0,86 & 1,00 & 0,97 & 0,86 & 1,00 & 1,00 & 0,90 & 1,00 \\
\hline 24 & 0,97 & 0,86 & 1,00 & 0,97 & 0,86 & 1,00 & 0,86 & 0,71 & 0,94 \\
\hline
\end{tabular}

\section{Análisis de los ítems}

En la tabla 2, se presenta que 23 ítems demuestran una distribución normal según el criterio de asimetría ( $\mathrm{g} 1<+/-1,5)$ y curtosis ( $\mathrm{g} 2<+/-3$ ), a diferencia del ítem 11, reportándose un índice de Mardia de 71,66, advirtiendo el incumplimiento de la propiedad de normalidad multivariada. 
Tabla 2.

Resumen de puntuaciones de los ítems del cuestionario

\begin{tabular}{|c|c|c|c|c|c|c|c|c|c|}
\hline \multicolumn{5}{|c|}{ Agresividad Premeditada } & \multicolumn{5}{|c|}{ Agresividad Impulsiva } \\
\hline Ítems & M & DS & g1 & g2 & Ítems & M & DS & g1 & g2 \\
\hline ítem 02 & 2,794 & 1,153 & 0,15 & $-0,889$ & ítem 01 & 2,912 & 1,241 & $-0,057$ & $-0,923$ \\
\hline ítem 04 & 3,884 & 0,957 & $-0,759$ & 0,324 & ítem 03 & 4,105 & 1,183 & $-1,296$ & 0,7 \\
\hline ítem 06 & 2,51 & 1,176 & 0,389 & $-0,693$ & ítem 05 & 3,674 & 1,005 & $-0,548$ & $-0,047$ \\
\hline ítem 08 & 2,61 & 1,272 & 0,277 & $-1,055$ & ítem 07 & 2,053 & 1,207 & 0,915 & $-0,157$ \\
\hline ítem 10 & 2,088 & 1,201 & 0,809 & $-0,483$ & ítem og & 2,951 & 1,208 & $-0,06$ & $-0,763$ \\
\hline ítem 12 & 2,443 & 1,22 & 0,469 & $-0,758$ & ítem 11 & 1,522 & 0,801 & 1,645 & 2,655 \\
\hline ítem 14 & 3,028 & 1,869 & $-0,025$ & $-1,874$ & ítem 13 & 2,689 & 1,308 & 0,14 & $-1,148$ \\
\hline ítem 16 & 1,976 & 1,094 & 0,911 & $-0,033$ & ítem 15 & 1,994 & 1,169 & 0,838 & $-0,472$ \\
\hline ítem 18 & 2,559 & 1,174 & 0,245 & $-0,85$ & ítem 17 & 2,161 & 1,176 & 0,614 & $-0,683$ \\
\hline ítem 20 & 2,176 & 1,11 & 0,697 & $-0,246$ & ítem 19 & 2,567 & 1,21 & 0,201 & $-1,041$ \\
\hline ítem 22 & 2,096 & 1,139 & 0,729 & $-0,469$ & ítem 21 & 2,386 & 1,251 & 0,469 & $-0,85$ \\
\hline ítem 23 & 3,011 & 1,268 & $-0,143$ & $-1,063$ & & & & & \\
\hline ítem 24 & 2,28 & 1,199 & 0,556 & $-0,7$ & & & & & \\
\hline
\end{tabular}

Nota: $g^{1}$ : Asimetría; $g^{2}$ : curtosis.

\section{Validez de estructura interna}

En la tabla 3, se presentan los índices de ajuste obtenidos por el método de mínimos cuadrados ponderados robustos [DWLS], siendo: $\mathrm{x2} / \mathrm{gl}=4,27, \mathrm{RSMEA}=, 064 \mathrm{~S}-\mathrm{RMR}=, 056$, $\mathrm{CFI}=, 96, \mathrm{TLI}=, 95$, indicando que el modelo ajusta.

Tabla 3.

Índices de ajuste del cuestionario

\begin{tabular}{cccccccc}
\hline Modelo & X2 & gl & x2/gl & $\begin{array}{l}\text { RSMEA } \\
(\text { IC90\% })\end{array}$ & SRMR & CFI & TLI \\
\hline 2 factores & 888,829 & 208 & 4,273 & $(, 060-, 068)$ & 0,056 & 0,956 & 0,952 \\
\hline
\end{tabular}

Nota: $x^{2}$ : Chi cuadrado; gl: Grados de libertad; RSMEA: Raíz cuadrada media del error de aproximación; SRMR: Raíz del residuo cuadrático media estandarizado; CFI: Índice de ajuste comparativo; TLI: Índice de Tucker-Lewis.

En la tabla 4, se muestran las cargas factoriales sobre el criterio aceptable en nueve de los 11 ítems del factor de agresividad impulsiva, en un rango entre ,36 a ,74, a diferencia de los ítems 3 y 5 que tuvieron cargas de -,25 y -,16; y el factor agresividad premeditada obtuvo cargas factoriales aceptables en 11 de sus 13 ítems con valores entre ,36 a ,71, a diferencia de los ítems 4 y 14 , con cargas factoriales de, 03 y, 22 respectivamente. 
Tabla 4 .

Cargas factoriales del cuestionario

\begin{tabular}{cccc}
\hline \multicolumn{2}{c}{ Agresividad Premeditada } & \multicolumn{2}{c}{ Agresividad Impulsiva } \\
\hline Ítems & $\lambda$ & Ítems & $\lambda$ \\
\hline ítem 02 & 0,57 & ítem 01 & 0,36 \\
ítem 04 & 0,03 & ítem 03 & $-0,25$ \\
ítem 06 & 0,64 & ítem 05 & $-0,16$ \\
ítem 08 & 0,71 & ítem 07 & 0,65 \\
ítem 10 & 0,61 & ítem 09 & 0,59 \\
ítem 12 & ítem 11 & 0,67 \\
ítem 14 & 0,58 & ítem 13 & 0,47 \\
ítem 16 & 0,22 & ítem 15 & 0,66 \\
ítem 18 & 0,67 & ítem 17 & 0,74 \\
ítem 20 & 0,36 & ítem 19 & 0,58 \\
ítem 22 & 0,68 & ítem 21 & 0,52 \\
ítem 23 & 0,69 & & \\
ítem 24 & 0,56 & & \\
\hline Cargas fact & 0,69 & &
\end{tabular}

Nota: $\lambda$ : Cargas factoriales

\section{Validez convergente}

En la tabla 5, se muestra la relación del CAPI-A con el RPQ, demostrando que existe correlación de magnitud grande entre las dimensiones del CAPI-A con la dimensión de agresividad reactiva $(r>50)$, y correlación de magnitud moderada entre las dimensiones del CAPI-A y la dimensión de agresión proactiva $(r>30)$, demostrando que ambos instrumentos miden constructos similares.

Tabla 5.

Correlación entre las variables del CAPI-A y el RPQ

\begin{tabular}{lccc}
\multicolumn{1}{c}{ Variables } & 1 & 2 & 3 \\
\hline 1. Agresión impulsiva & & & \\
2. Agresión premeditada & 0,51 & 0,60 & \\
3. Agresividad reactiva & 0,47 & 0,42 & 0,54 \\
4. Agresividad proactiva & & & \\
\end{tabular}

Nota: La correlación entre los dos instrumentos (CAPI-A - RPQ) se encuentra fuera de los triángulos.

\section{Confiabilidad}

En la tabla 6, se muestra la consistencia interna obtenida por el coeficiente Omega de ,79 para AP y de ,73 para Al, consideradas como aceptables. Corroborando que los ítems miden homogéneamente un concepto similar, por tanto, ofrecen puntuaciones consistentes. Así también, se presentan las puntuaciones del análisis de estabilidad temporal. Con una 
segunda aplicación del instrumento en estudiantes $(n=200)$, se obtuvo correlaciones testretest de magnitud grande $\left(r_{\text {test-restes }}=, 70\right)$ en ambas dimensiones, concluyendo que las mediciones del cuestionario no varían en el tiempo, garantizando puntuaciones estables.

Tabla 6.

Consistencia interna por el coeficiente de Omega del cuestionario

\begin{tabular}{lccc}
\hline \multicolumn{1}{c}{ Factor } & Ítems & $\omega$ & $r_{\text {test-restes }}$ \\
\hline Agresividad Premeditada & 13 & 0,79 &, 75 \\
Agresividad Impulsiva & 11 & 0,73 &, 70 \\
\hline
\end{tabular}

Nota: $\omega$ : Coeficiente de consistencia interna. $r_{\text {test-restes: }}$ Coeficiente de correlación.

\section{Análisis de la agresividad premeditada e impulsiva}

Las puntuaciones obtenidas por los escolares en la evaluación de la agresividad premeditada e impulsiva evidencian diferencias significativas entre varones y mujeres en ambas dimensiones. Siendo los rangos promedios mayores en los escolares del género masculino, lo que marca una tendencia en el ejercicio de la agresión tanto premeditada como impulsiva en los escolares varones en comparación a las mujeres.

Tabla 7.

Diferencias en la agresividad premeditada e impulsiva en la muestra de escolares

\begin{tabular}{|c|c|c|c|c|c|c|c|c|}
\hline Agresividad & Género & $\mathrm{N}$ & $\begin{array}{c}\text { Rango } \\
\text { promedio }\end{array}$ & $\begin{array}{c}\text { Suma de } \\
\text { rangos }\end{array}$ & $\begin{array}{l}\text { U de Mann- } \\
\text { Whitney }\end{array}$ & $\begin{array}{c}\text { W de } \\
\text { Wilcoxon }\end{array}$ & Z & $\mathrm{p}$ \\
\hline \multirow{2}{*}{ Premeditada } & Masculino & 280 & 290,93 & 60513,50 & \multirow{2}{*}{28822,500} & \multirow{2}{*}{81797,500} & \multirow{2}{*}{$-2,872$} & \multirow{2}{*}{0,004} \\
\hline & Femenino & 248 & 251,68 & 81797,50 & & & & \\
\hline \multirow[b]{2}{*}{ Impulsiva } & Masculino & 280 & 296,09 & 61587,00 & \multirow{2}{*}{27749,000} & \multirow{2}{*}{80724,000} & \multirow{2}{*}{$-3,493$} & \multirow{2}{*}{0,000} \\
\hline & Femenino & 248 & 248,38 & 80724,00 & & & & \\
\hline
\end{tabular}

\section{Discusión y conclusiones}

La agresividad en adolescentes es una problemática manifestada en diversas investigaciones (Caballero et al., 2018; Martín Montilla et al., 2016; Zuiev, 2016;), de la misma forma que en las estadísticas sobre el acoso escolar (Unesco, 2018). Por ello, es necesario que los docentes posean herramientas útiles que les posibiliten identificar casos de agresividad, para posteriormente actuar. En tal sentido, el CAPI-A representa una herramienta útil para cuantificar la agresividad y luego generar intervenciones objetivas. Por este motivo, tal investigación realizó una revisión psicométrica del cuestionario CAPI-A.

Mediante el criterio de jueces se reportaron índices aceptables de relevancia, coherencia y claridad (Vo > ,70; Charter, 2003), ello implica poseer enunciados representativos para medir el constructo de la agresividad en sus dimensiones impulsiva y premeditada; conteniendo enunciados que pueden comprenderse y guardan relación con el concepto teórico. Además, 
se corroboró que cada ítem se sostiene en función a la conducta, pues, de esta forma se definen sin ambigüedad y son relevantes para el constructo medido.

El análisis factorial confirmatorio, mediante el método de mínimos cuadrados ponderados robustos, corroboró el modelo empírico con el teórico, obteniendo valores aceptables de los índices de ajuste x2/gl, RSMEA, S-RMR, CFI y TLI (Ríos y Wells, 2014); además de las cargas factoriales aceptables de 20 de los ítems del CAPI-A que superan el punto de corte (Hair et al., 2004), excepto los ítems 3, 4, 5 y 14; corroborando la idoneidad del modelo de dos factores. Como se mencionó anteriormente, estos resultados demuestran la idoneidad del modelo de dos dimensiones y la pertinencia de los ítems en la representatividad del factor que mide agresividad impulsiva sobre respuestas instintivas y emocionales, derivadas de una provocación de la víctima o un tercero; y el factor de agresividad premeditada sobre la conducta como un medio para obtener un objetivo diferente a dañar a la víctima, coacción o defensa del propio poder, domino o estatus social (Andreu, Peña y Ramírez, 2009).

También se investigó la correlación el CAPI-A con un test de constructo similar, el RPQ. Anteriormente, mediante la validez convergente estudiada por Andreu, Peña y Larroy (2010) obtuvieron resultados favorables. En este estudio se encontró que existe correlación de magnitud grande (Cohen, 1988) entre las dimensiones CAPI-A con la modalidad de agresividad reactiva del RPQ $(r>, 50)$ y relación de magnitud moderada (Cohen, 1988) entre las dimensiones CAPI-A con la agresión proactiva del RPQ $(r>30)$ en adolescentes del distrito de Trujillo. De esta forma se respalda el uso del cuestionario y demuestra que ambos instrumentos miden constructos similares relacionados a la agresividad, la agresividad reactiva e impulsiva como componente primitivo e instintivo resultante de la provocación; y la agresión premeditada y proactiva como respuesta que busca un beneficio para el autor de la agresión; las cuatro, manifestaciones conductuales originados de procesos mentales (Andreu, Peña y Larroy, 2010).

La consistencia interna reporta valores aceptables hallados a través del coeficiente de Omega, mismos que están sobre el punto de referencia (Ventura-León, 2018). De esta forma, se corrobora un indicador de que los ítems miden homogéneamente un concepto similar y no presentan influencia considerable del error (Brown, 2006). Además, por medio del método test-retest (Aldridge et al., 2017) se determinó correlaciones significativas y se concluyó que las calificaciones de las dimensiones del CAPI-A son estables a través del tiempo. Estos resultados concuerdan con otros estudios (Andreu, Peña y Ramírez, 2009) y sugieren la pertinencia de la replicabilidad de los datos encontrados a población con características similares (Gempp Fuentealba, 2006).

En respuesta al objetivo descriptivo comparativo del estudio, se evidenció diferencias en la agresividad premeditada e impulsiva. El hallazgo demuestra que los escolares de género masculino son más propensos a justificar y emplear la agresión como herramienta para concretar sus objetivos, además de dominar y ejercer poder sobre los demás. Respecto a ello, Finigan-Carr et al. (2016) concuerdan y reportan mayores puntuaciones en agresión instrumental en los varones; observación respaldada en la idea de que los hombres adoptan este modelo de conductas para obtener poder y autoridad. Del mismo modo, las puntuaciones mayores en la agresión impulsiva, señalan que los varones tienden a mostrar respuestas agresivas no planificadas, propensión a interpretar las situaciones sociales como intencionalmente hostiles y menores niveles de reflexión; en comparación a las escolares mujeres (Andreu, 2010). Otros estudios presentaron resultados similares; por ejemplo, Muñoz-Reyes et al. (2016) y Björkqvist (2018) identificaron que los varones muestran mayores niveles de agresividad física que las mujeres. 
En el ámbito educativo, la educación para la convivencia constituye una prioridad dadas las elevadas tasas de violencia desarrolladas en nuestro país. Por lo que, la escuela puede conducirse en un marco de respeto por los derechos humanos, creando relaciones interpersonales saludables (Villamizar-Ibarra, 2016), hecho que posibilitará un mejor proceso enseñanza-aprendizaje. Conocer y comprender la agresividad en las escuelas, es esencial como punto de partida para el diseño y la implementación de programas que prevengan o atiendan casos de violencia escolar (Pacheco-Salazar, 2018).

Las medidas que pueden adoptarse están vinculadas a intervenciones enfocadas en promover normas prosociales, motivar a la conducta positiva y organizar aspectos del aula como la disposición de los grupos y de los asientos (Thomas et al., 2011). No obstante, previo a la implementación de programas, tanto de promoción como de prevención, es relevante una evaluación previa que facilite identificar el grado de la problemática, incluso, la diferenciación de los grupos de educandos que merecen mayor atención. En este caso, el CAPI-A es una herramienta potencial con la que el docente puede contar. Otro beneficio que proporcionan los instrumentos de medición, es la utilización de estas escalas para cuantificar los efectos de sus intervenciones.

En paralelo, tal y como señala Rubio Hernández et al. (2019), enfocado en la detección y prevención del bullying y cyberbullying, es fundamental la incorporación de la familia y otras instituciones necesarias para disminuir las altas tasas de violencia escolar. Como señala Cuenca Sánchez y Mendoza González (2017), los padres representan agentes efectivos para disminuir comportamientos agresivos y aumentar conductas pro sociales.

Otros actores que pueden ser útiles en la disminución de esta problemática, son los consejeros escolares, quienes poseen un papel principal en la prevención de la violencia, capacitando a los docentes en habilidades básicas de consejería para interactuar de forma adecuada con los estudiantes (Volungis y Goodman, 2017). Ello con el propósito de ayudar a motivar un clima de confianza, dado que se ha constatado que cuando existen relaciones sólidas, en un ambiente adecuado, entre maestros y alumnos, los educandos se hallan más dispuestos a comunicar cuando tienen conocimiento de algún posible acto violento (Volungis y Goodman, 2017).

Los resultados hallados en la investigación son significativos; sin embargo, existen limitaciones como la clase de muestreo no probabilístico y la muestra utilizada con sujetos que pertenecen a una misma institución educativa. Por lo tanto, se sugiere emplear un muestreo probabilístico e implementar validez en función de la respuesta en los ítems, asimismo estudiar el cuestionario en otros contextos y con otros diseños y propósitos.

Por último, se evidencia de validez de contenido, de estructura, convergente y adecuada confiabilidad del CAPI-A en la muestra de escolares. Además, los escolares de género masculino muestran puntuaciones notablemente distintas a las conseguidas por las de género femenino, tanto en agresión premeditada como impulsiva. La contribución más beneficiosa, y por ello valiosa, de este estudio es presentar el CAPI-A como una escala útil para la medición y análisis de este constructo, que puede emplearse tanto en la práctica profesional como en el desarrollo de posteriores investigaciones interesadas en la detección de conductas agresivas dentro del salón de clases. 


\section{Referencias}

Aldridge, V. K., Dovey, T. M. y Wade, A. (2017). Assessing Test-Retest Reliability of Psychological Measures Persistent Methodological Problem. European Psychologist, 22(4), 207-218. https://doi.org/10.1027/1016-9040/a000298

Álvarez-García, D., Rodríguez, C., González-Castro, P., Núñez, J. C. y Álvarez, L. (2010). La formación de los futuros docentes frente a la violencia escolar. Revista de Psicodidáctica, 15(1), 35-56.

Andreu, J. M. (2010). Manual del Cuestionario de agresividad premeditada e impulsiva en adolescentes CAPI-A. TEA.

Andreu, J. M., Peña, M. E. y Larroy, C. (2010). Conducta antisocial, impulsividad, y creencias justificativas: análisis de su interrelación con la agresión proactiva y reactiva en adolescentes. Psicología Conductual: Revista Internacional Clínica y de la Salud, 18(1),5772.

Andreu, J. M., Peña, M. E. y Ramírez, J. M. (2009). Cuestionario de agresión reactiva y proactiva: instrumento de medida de la agresión en adolescentes. Revista de psicopatología y psicología clínica, 14(1), 37-49.

Andreu Rodríguez, J. M., Ramírez, J. M. y Raine, A. (2006). Un modelo dicotómico de la agresión: valoración mediante dos autoinformes (CAMA y RPQ). Psicopatología Clínica Legal y Forense, 6(1-3), 25-42.

Ato, M., López-García, J. J. y Benavente, A. (2013). Un sistema de clasificación de los diseños de investigación en psicología. Anales de Psicología, 29(3), 1038-1059. http://dx.doi.org/10.6018/analesps.29.3.178511

Björkqvist, K. (2018). Gender differences in aggression. Currente Opinion in Psychology, 19, 3942. https://doi.org/10.1016/j.copsyc.2017.03.030

Boboc, D. y Rosoiu, N. (2019). Aggressiveness in Children - A Serious Problem of Nowadays' Society. Annals Series on Biological Sciences, 8(1), 53-64.

Brown, T. (2006). Confirmatory factor analysis for applied research. Guildford Press.

Caballero, S. V., Contini de Gonzalez, N., Lacunza, A. B., Mejail, S. y Coronel, P. (2018). Habilidades sociales, comportamiento agresivo y contexto socioeconómico. Un estudio comparativo con adolescentes de Tucumán (Argentina). Cuadernos de la Facultad de Humanidades y Ciencias Sociales - Universidad Nacional de Jujuy, (53), 183203.

Charter, R. A. (2003). A breakdown of reliability coefficients by test type and reliability method, and the clinical implications of low reliability. The Journal of General Psychology, 130(3), 290-304. https://doi.org/10.1080/00221300309601160

Cohen, J. (1988). Statistical power analysis for the behavioral sciences (2nd ed.). Lawrence Earlbaum Associates.

Cuenca Sánchez, V. y Mendoza González, B. (2017). Comportamiento prosocial y agresivo en niños: tratamiento conductual dirigido a padres y profesores. Acta de investigación psicológica, 7(2). https://doi.org/10.1016/j.aipprr.2017.03.005

Denson, T. F., O’Dean, S. M., Blake, K. R. y Beames, J. R. (2018). Aggression in Women: Behavior, Brain and Hormones. Frontiers in Behavioral Neuroscience, 12. https://doi.org/10.3389/fnbeh.2018.00081 
Escobedo Portillo, M.T., Hernández Gómez, J. A., Estebané Ortega, V. y Martínez Moreno, G. (2016). Modelos de ecuaciones estructurales: características, fases, construcción, aplicación y resultados. Ciencia \& trabajo, 18(55), 16-22. http://dx.doi.org/10.4067/S0718-24492016000100004

Farnicka, M. y Grzegorzewska, I. (2015). Intrapersonal correlates of aggression in adolescents: determinants of undertaking the roleof the perpetrator and the victim. Current issues in personality psychology, 3(1), 25-35. https://doi.org/10.5114/cipp.2015.49940

Finigan-Carr, N. M., Gielen, A., Haynie, D. L., y Cheng, T. L. (2016). Youth Violence. Journal of Interpersonal $\quad$ Violence, 31(19), 3257-3281. https://doi.org/10.1177\%2F0886260515584348

Fondo de las Naciones Unidas para la Infancia. (2018). Una lección diaria. Acabar con la violencia en las escuelas \#ENDViolence. Nueva York.

Garaigordobil, M., Martínez-Valderrey, V. y Machimbarrena, J. M. (2017). Intervención en el bullying y cyberbullying: Evaluación del caso Martín. Revista de Psicología Clínica con Niños y Adolescentes, 4(1),25-32.

Gempp Fuentealba, R. (2006). El error estándar de medida y la puntuación verdadera de los tests psicológicos: Algunas recomendaciones prácticas. Terapia Psicológica, 24(2), 117129.

Hair, J., Anderson, R., Tatham, R. y Black, W. (2004). Análisis Multivariante (5ta Ed.). Prentice Hall.

Jiménez-Barbero, J. A., Ruiz-Hernández, J. A., Llor-Esteban, B. y Waschgler, K. (2016). Influence of attitudes, impulsivity, and parental styles in adolescents' externalizing behavior. Journal of Health Psychology, 21(1), 122-131. https://psycnet.apa.org/doi/10.1177/1359105314523303

Martín Montilla, A., Pazos Gómez, M., Montilla Coronado, M. C. y Romero Oliva, C. (2016). Una modalidad actual de violencia de género en parejas de jóvenes: las redes sociales. Educación XX1, 19(2), 405-429. http://dx.doi.org/10.5944/educxx1.16473

Martínez Martínez, A., Ruiz-Rico Ruiz, G., Zurita Ortega, F., Chacón Cubreros, R., Castro Sánchez, M. y Cachón Zagalaz, J. (2017). Actividad física y conductas agresivas en adolescentes en régimen de acogimiento residencia. Suma Psicológica, 24(2), 135-141. https://doi.org/10.1016/j.sumpsi.2017.02.002

McMahon, S. D., Peist, E., Davis, J. O., Bare, K., Martinez, A., Reddy, L. A., Espelage, D. L. y Anderman, E. M. (2019). Physical aggression toward teachers: Antecedents, behavior and consequences. Aggressive Behavior, 4(1), 116-126. https://doi.org/10.1002/ab.21870

Muñoz-Reyes, J. A., Polo, P., Valenzuela, N., Guerra, R., Anabalón, K., Hidalgo-Rasmussen, C., y Turiégano, E. (2016). Sexual Differences and Associations between Aggressiveness and Quality of Life in Late Adolescents. Current Psychology, 37(1), 28-37. https://doi.org/10.1007/s12144-016-9486-3

Organización de las Naciones Unidas para la Educación, la Ciencia y la Cultura. (2018). School violence and bullying: Global status and trends drivers and consequences. París: UNESCO.

Pacheco-Salazar, B. (2018). Violencia escolar: la perspectiva de estudiantes y docentes. Revista electrónica de investigación educativa, 20(1), 112-121. 
Perez Bernardes de Moraes, T. (2013). ¿Por qué los hombres presentan un comportamiento más agresivo que las mujeres? Por una antropología evolutiva del comportamiento agresivo. Nómadas: Critical Journal of Social and Juridical Sciences, (37), 93-111.

Pinazo, D., García-Prieto, L. T. y García-Castellar, R. (2020). Implementation of a program based in mindfulness for the reduction of aggressiveness in the classroom. Revista Psicodidáctica (English ed.), 25(1), 30-35. https://doi.org/10.1016/j.psicoe.2019.08.003

Raine, A., Dodge, K., Loeber, R., Gatzke-Kopp, L., Lynam, D., Reynolds, C., StouthamerLoeber, M. y Liu, J. (2006). The reactive-proactive aggression questionnaire: Differential correlates of reactive and proactive aggression in adolescent boys. Aggressive Behavior, 32(2), 159-171. https://doi.org/10.1002/ab.20115

Ruiz-Hernández, J. A., Moral-Zafra, E., Llor-Esteban, B., y Jiménez-Barbero, J. A. (2019). Influence of parental styles and other psychosocial variables on the development of externalizing behaviors in adolescents: A sytematic review. The European Journal of Psychology Applied to Legal Context, 11(1), 9-21. https://psycnet.apa.org/doi/10.5093/ejpalc2018a11

Ríos, J. y Wells, C. (2014). Validity evidence based on internal structure. Psicothema, 26(1), 108116. https://doi.org/10.7334/psicothema2013.260

Rojas Zegarra, M. E., Arias Gallegos, W. L., Rivera, R., Geldres García, J. A., Starke Moscoso, M. A. y Apaza Bejarano, E. N. (2020). Propiedades psicométricas de los cuestionarios Reactive/Proactive Questionnaire (RPQ) y How I Think Questionaire (HIT) en estudiantes peruanos. Revista de Psicopatología y Psicología Clínica, 25(1), 59-68.

Rubio Hernández, F. J., Díaz López, A. y Cerezo Ramírez, F. (2019). Bullying y cyberbullying: la respuesta de las comunidades autónomas. Revista Electrónica Interuniversitaria de Formación del Profesorado, 22(1), 145-157. https://doi.org/10.6018/reifop.22.1.332311

Ruíz, M. A., Pardo, A. y San-Martín, R. (2010). Modelo de ecuaciones estructurales. Papeles del psicólogo, 31(1), $34-45$.

Sireci, S. y Faulkner-Bond, M. (2014). Validity evidence based on test content. Psicothema, 26(1), 100-107.

Socastro-Gómez, A. y Jiménez, A. (2019). Agresividad impulsiva y proactiva, moldes mentales y rasgos de personalidad en adolescentes. Behavior \& Law Journal, 5(1), 31-39. https://doi.org/10.47442/blj.v5.11.66

Thomas, D. E., Bierman, K. L. y Powers, C. J. (2011). The Influence of Classroom Aggression and Classroom Climate on Aggressive-Disruptive Behavior. Child Development, 82(3), 751-757. https://dx.doi.org/10.1111\%2Fj.1467-8624.2011.01586.x

Volungis, A. M. y Goodman, K. (2017). School Violence Prevention: Teachers Establishing Relationships With Students Using Counseling Strategies. SAGE Journals, 7(1). https://doi.org/10.1177\%2F2158244017700460

Ventura-León, J. L. (2018). Intervalos de confianza para coeficiente Omega: Propuesta para el cálculo. Adicciones, 30(1), 77-78.

Villamizar-Ibarra, J. (2016). Pedagogía para la convivencia y la paz: transformaciones que experimentan los docentes cuando vivencian la formación para la convivencia y la paz. Aibi Revista de Investigación, Administración e Ingeniería, 4(2), 56-62.

Zuiev, I. (2016). Studying Of Young Criminals' Aggression With The Help Of various Methods. The Journal of V. N. Karazin Kharkiv National University. A Series of «Psychology», (60), 62-65. 